\title{
Conductive Hearing Loss Results in Changes in Cytochrome Oxidase Activity in Gerbil Central Auditory System
}

\author{
Debara L. Tucci, ${ }^{1}$ Nell B. Cant, ${ }^{2}$ and Dianne Durham ${ }^{3}$ \\ ${ }^{1}$ Division of Otolaryngology-Head and Neck Surgery, Duke University Medical Center, Durham, NC 27710, USA \\ ${ }^{2}$ Department Neurobiology, Duke Universtiy Medical Center, Durham, NC 27710, USA \\ ${ }^{3}$ Department of Otolaryngology, Universtiy of Kansas Medical Center, Kansas City, KS 66160, USA
}

Received: 21 November 2000; Accepted: 17 July 2001; Online publication: 31 August 2001

\begin{abstract}
Conductive hearing loss (CHL) restricts auditory input to an intact peripheral auditory system. Effects of deprivation on the central auditory system (CAS) have been debated, although a number of studies support the hypothesis that CHL can cause modification of CAS structure and function. The present study was designed to test the hypothesis that unilateral CHL results in a decrease in cytochrome oxidase $(\mathrm{CO})$ activity in CAS nuclei that receive major afferent input from the affected ear. Gerbils at postnatal day 12 (P21) or 6-8 weeks underwent left unilateral CHL (malleus removal), cochlear ablation, or a sham surgical procedure. After a survival time of 48 hours or 3 weeks, animals were sacrificed and tissue was processed for cytochrome oxidase histochemistry. Optical density (OD) measurements were made from individual neurons in the anteroventral cochlear nucleus (AVCN) and from medial and lateral dendritic fields in the medial superior olivary nucleus (MSO), the lateral superior olivary nucleus, and the inferior colliculus. The width of the CO-stained neuropil in MSO was also measured as an estimate of dendritic length. OD measures were corrected to neutral areas of the brain. Cochlear ablation caused significant decreases in $\mathrm{CO}$ activity in left lower brainstem nuclei, particularly in adult animals. Following CHL, a significant decrease in CO activity was observed in the ipsilateral AVCN and a significant increase was observed in the contralateral
\end{abstract}

Correspondence to: Dr. Debara L. Tucci - Department of Surgery • Division of Otolaryngology-Head and Neck Surgery • Duke University Medical Center - Box 3805 - Durham, NC 27710. Telephone: (919) 684-6968; fax: (919) 681-6881; email: tucci001@mc.duke.edu
AVCN. Cochlear ablation resulted in decreased width of MSO neuropil containing dendrites that receive primary input from the ablated ear. CHL resulted in a significant increase in the width of MSO neuropil on both sides of the brain in the P21 animals that survived 3 weeks but not in P21 animals that survived only 48 hours or in the adult animals. Unilateral CHL is associated with changes in $\mathrm{CO}$ activity in the AVCN and may affect MSO dendritic length in younger animals.

Keywords: deprivation, plasticity, brain metabolism

\section{INTRODUCTION}

The long-term effects of chronic conductive hearing impairment on central auditory system (CAS) structure and function are not well known. This is in contrast to the well-documented effects of sensorineural hearing loss (SNHL), which are modeled in animal studies of auditory system deafferentation. Cochlear removal or damage results in a dramatic decrease in auditory nerve activity (Koerber et al. 1966; Born et al. 1991). Degenerative changes in the auditory brainstem, including a loss of neurons and a reduction in neuron size (Powell and Erulkar 1962; Trune 1982a, b; Nordeen et al. 1983a; Born and Rubel 1985; Pasic and Rubel 1989; Hashisaki and Rubel 1989; Tierney et al. 1997), protein synthesis (Born and Rubel 1988; Hyson and Rubel 1989; Sie and Rubel 1992), and oxidative enzyme activity (Durham and Rubel 1985; Durham et al. 1993; Hyde and Durham 1990), are thought to occur secondary to a loss of excitatory synaptic activity. 
In contrast, conductive hearing loss (CHL) has been shown in one experimental paradigm to have no significant effect on spontaneous or baseline auditory nerve activity (Tucci et al. 1987), although this condition results in a clear reduction in sound-evoked activity. Studies by Webster and colleagues (Webster and Webster 1977, 1979; Webster 1983a, b, c, 1988) demonstrated that early removal of the ear canal blastema in the immature mouse results in a significant decrease in CAS neuron areas, as long as the manipulation falls within a critical period of 12-24 days after birth. Further studies in the mouse by Trune and colleagues (Trune and Morgan 1988a, b; Trune and Kiessling 1988) demonstrated changes in the cochlear nucleus following unilateral CHL. Coleman et al. (Coleman and O'Connor 1979; Coleman et al. 1982; Blatchley et al. 1983) also reported age-dependent effects of unilateral CHL in rat, with decreased area of spherical cells in the anteroventral cochlear nucleus (AVCN) and decreased volume in the dorsal (DCN) and ventral (VCN) cochlear nucleus. Conlee and Parks (1981) produced a unilateral CHL by earplug in an embryonic chick and found a significant progressive decrease in size of second-order auditory neurons by 25 and 60 days after hatching.

Several studies have focused on the effect of unilateral CHL on morphology of dendrites in the medial superior olive (MSO), the first nucleus in the mammalian brain to receive binaural input, or its avian homo$\log$, n. laminaris (NL). These third-order neurons receive segregated dendritic inputs, with afferent input from each ear projecting to the ipsilateral and contralateral MSO. Feng and Rogowski (1980) reported that placement of a unilateral earplug at the time of ear canal opening (postnatal day 12, P12) resulted in restricted growth of the MSO dendrites that receive afferent input from the effected ear. Similar results were reported following placement of unilateral earplugs in embryonic chicks (Gray et al. 1982; Smith et al. 1983; Conlee and Parks 1983).

Conversely, Moore and colleagues (Moore et al. 1989; Moore 1991) have demonstrated in the ferret that a neonatally induced unilateral CHL (produced before hearing ability reaches adultlike maturity) does not result in changes in neuron size in the CN. It is, however, associated with a significant alteration in an auditory brainstem pathway, with an increase in the number of $\mathrm{CN}$ neurons contralateral to the hearing loss ear projecting to its ipsilateral inferior colliculus (IC). This ipsilateral CN-IC projection, which is normally quite small in comparison with the primary contralateral projection, increases by an average 28\% following unilateral CHL. Other studies that failed to find an effect of unilateral CHL in the $\mathrm{CN}$ neuron area include a study by Tucci and Rubel (1985) in the chick and one by Doyle and Webster (1991) in the rhesus monkey.

Development of binaural hearing may be uniquely affected by a unilateral decrease in afferent input. Studies by Clopton and Silverman (1977, 1978; Silverman and Clopton 1977) that used rats raised with unilateral conductive hearing loss demonstrated altered binaural interaction properties in the IC. Altered binaural response properties were reported following monaural ligation in cats, as measured in IC by Moore and Irvine (1981) and in auditory cortex by Brugge et al. (1985). Knudsen and colleagues (reviewed in Knudsen 1999) have documented extensively the plasticity of auditory localization pathways following monaural occlusion in the barn owl. Based on information gleaned from these studies and others, including studies in the human auditory system (Hall and Derlacki 1988; Pillsbury et al. 1991; Wilmington et al. 1994; Hall et al. 1995), it is likely that monaural deprivation, as produced by conductive hearing loss, produces structural and functional changes in the CAS that are both greater than those produced by bilateral CHL and possibly different in nature than unilateral deafferentation, such as with end-organ ablation.

In a recent study (Tucci et al. 1999), we demonstrated in the gerbil that unilateral CHL results in a marked decrease in the uptake of ${ }^{14} \mathrm{C}$-2-deoxyglucose in the major afferent projection from an ear with a CHL. The effect of ossicle removal, which produced a CHL, and the effect of cochlear ablation (CA), which produced complete deafferentation of the auditory system, were quite similar, particularly in young (21 day old, P21) animals. The 2-DG method reflected synaptic activity in the CAS at the time the animal was sacrificed. In order to determine whether there are long-term changes in auditory system function, we studied cytochrome oxidase $(\mathrm{CO})$ activity in the CAS following unilateral CHL. CO activity in second-order auditory system neurons has been demonstrated to decrease significantly following deafferentation by cochlea removal (Wong-Riley et al. 1978; Hyde and Durham 1990). The purpose of this investigation was to determine if unilateral CHL also results in changes in $\mathrm{CO}$ activity in brainstem CAS neurons. Individual neuron measurements were made in the rostral region of AVCN in order to include spherical bushy cells which project to the MSO. Whole nucleus measurements were made in MSO, LSO, and selected portions of the IC. A secondary goal of this investigation was to assess changes in the length of the segregated dendritic fields of the MSO, as these have been demonstrated in previous studies to be affected by both unilateral cochlear ablation and CHL. Dendritic length was approximated using an indirect measure, the width of the CO-stained neuropil medial and lateral to the dorsoventral axis of the MSO. 
It has been suggested that structural and functional changes in CAS neurons occur as a result of significant alterations in auditory nerve activity (Rubel et al. 1990). Since CHL is thought to produce changes in sound-evoked but not baseline, or spontaneous, auditory nerve activity, a finding of a decrease in enzymes related to oxidative metabolism in these neurons may indicate that the amount and type of sum total auditory nerve activity, and not just the presence or absence of normal spontaneous activity, is important in maintaining normal CAS function. Based on the results of our 2-DG studies, we hypothesized that neurons that receive input from an ear with a conductive hearing loss would demonstrate decreased CO activity when compared with neurons that receive normal auditory input.

\section{METHODS}

\section{Subjects}

Gerbils (Meriones unguiculatus) served as subjects for this experiment. Animals were obtained from Tumblebrook Farms, Inc. (West Brookfield, MA, USA) at the appropriate ages. Younger (P21) animals were shipped with their mothers but were housed separately after the surgical procedure. Animals were housed in an animal facility approved by the American Association for Accreditation of Laboratory Animal Care, and the experimental protocol was reviewed and approved by the appropriate Institutional Animal Care and Use committees.

\section{Experimental design}

Independent variables were age, survival time, and type of surgical procedure: cochlear ablation, malleus removal, and sham procedure (all unilateral). The dependent variables were optical density of $\mathrm{CO}$ reaction product in the brainstem auditory nuclei and measures of the width of CO-stained neuropil in the medial superior olivary complex (MSO) of experimental vs. sham animals. Three age/survival groups were used for each of three experimental conditions (Table 1). Animals were entered into the study at postnatal day 21 (P21; "juveniles") or at 6-8 weeks ("adult").

Gerbils at P21 have fully formed ossicles and a wellaerated middle ear space. Single-unit responses in the ventral cochlear nucleus (VCN) are adultlike by P18 but auditory responses continue to mature until at least P30 (Ryan 1976; Woolf and Ryan 1984, 1985). Thus, animals at P21 have mature auditory thresholds and an anatomically mature middle and inner ear but still undergo active developmental changes in the function of the CAS. The juvenile group (P21) was studied

\begin{tabular}{lccc}
\hline \multicolumn{4}{c}{ TABLE $\mathbf{1}$} \\
\hline \multicolumn{4}{c}{ Animal treatment groups and numbers of subjects } \\
\hline Animal group & $C H L^{\mathrm{a}}$ & $\mathrm{CA}^{\mathrm{b}}$ & $\mathrm{SH}^{\mathrm{c}}$ \\
\hline Juvenile (48 hours) & 8 & 5 & 5 \\
Juvenile (3 week) & 7 & 6 & 7 \\
Adult (3 week) & 9 & 5 & 8 \\
\hline${ }^{\mathrm{a}} \mathrm{CHL}=$ conductive hearing loss. \\
${ }^{\mathrm{b}} \mathrm{CA}=$ cochlear ablation. \\
${ }^{\mathrm{C}} \mathrm{SH}=$ sham.
\end{tabular}

for each experimental condition at two survival times: 48 hours and 3 weeks after onset of hearing loss. Adult animals were studied 3 weeks after surgical manipulation.

Animals in each age/survival group underwent one of three unilateral (left ear) surgical manipulations: CHL produced by malleus removal, sensorineural hearing loss produced by cochlear ablation (CA), or a sham procedure.

\section{Surgical procedure}

All surgical procedures were performed unilaterally, always on the left ear. Animals were anesthetized with a combination of ketamine $[75 \mathrm{mg} / \mathrm{kg}$ intraperitoneally (IP)] and xylazine ( $5 \mathrm{mg} / \mathrm{kg} \mathrm{IP})$ given as a mixture. Additional anesthetic was administered throughout the procedure at a fraction of the original dose as needed to maintain anesthesia. Adult animals were supplemented with $0.20 \mathrm{~mL}$ of $1 \%$ lidocaine with 1:100,000 epinephrine at the incision site as a local anesthetic.

A postauricular incision was made. For sham animals, the procedure was terminated at this point and the wound closed. For the CHL and CA groups, soft tissue dissection was carried out along the posterior cartilaginous ear canal to the bony cartilaginous junction and the ear canal skin incised. The tympanic membrane was visualized and punctured with jeweler's forceps, and the malleus was removed through this opening. The stapes was visualized to insure stability within the oval window; malleus removal did not disrupt the stapes in any case. For the CHL group, the procedure was terminated at this point and the wound closed. For the CA group, following malleus removal the lateral wall of the cochlea was fractured with forceps and a large segment of the wall was removed. Suction was used to aspirate cochlear fluid and membranous components. The wound was then closed with suture or cyanoacrylate glue.

Animals recovered under supervision and were returned to their cages when fully awake. They were maintained in the animal facility with full access to food and water for the appropriate survival time. 


\section{Tissue processing}

At the appropriate survival time, animals were injected with ${ }^{14} \mathrm{C}$-2-deoxyglucose (2-DG) (for a companion study, see Tucci et al. 1999) and exposed to laboratory sounds for 45 minutes. Following halothane inhalation anesthesia, animals were decapitated and brains were quickly removed, frozen in heptane cooled in dry ice to $-65^{\circ} \mathrm{C}$, and then stored in closed vials at $-80^{\circ} \mathrm{C}$ until cutting. Brains were notched at the right cerebellum, and transverse sections through the brainstem auditory nuclei were cut at 16- $\mu \mathrm{m}$ thickness on a cryostat at $-21^{\circ} \mathrm{C}$. A one-in-four series of sections was rapidly thaw-mounted onto Superfrost Plus slides (Fisher Scientific, St. Louis, MO), air dried for at least 30 minutes, and then processed within 6 hours of sectioning using an established $\mathrm{CO}$ protocol (Hyde and Durham 1990). Slides were incubated at $37^{\circ} \mathrm{C}$ in a staining solution which contained, per $100 \mathrm{~mL}$, the following: $50 \mathrm{mg}$ diaminobenzidine, $20 \mathrm{mg}$ cytochrome C, type III (Sigma Chemical Co., St. Louis, MO, USA), and $4 \mathrm{~g}$ sucrose in $0.1 \mathrm{M}$ phosphate buffer $(\mathrm{PB})$ at $\mathrm{pH}$ 7.4. After a 60-90-min incubation, the sections were washed in four changes of $\mathrm{PB}$ to stop the reaction and then dehydrated in graded alcohols and coverslipped with DPX.

\section{Tissue analysis}

Sections that spanned the anterior-to-posterior dimension of each individual auditory brainstem nucleus on both the left and right sides of the brain were identified. Optical density (OD) measurements were obtained using the National Institutes of Health computerized image analysis software (NIH Image). Images were captured through the microscope with a MTI CCD725 video camera and digitized by a frame grabber board housed in a Macintosh 7100 computer. Calibration was performed using an internal standard to convert the gray level values obtained by the camera to optical density values. For each set of auditory nucleus measurements, an unaffected or "neutral" area of the brain was selected and measured to control for any intraanimal differences unrelated to the function of the central auditory system. Measurements were taken in the following locations:

1. AVCN: Individual OD measurements were made of

FIG. 1. A Neurons in rostral region of AVCN. B MSO was bisected along the line of cell bodies, and measurements of $\mathrm{CO}$ optical density and neuropil width were made medial and lateral to this line in the dorsal, middle, and ventral regions of the nucleus. C Optical density measurements were made in the dorsal, ventral, and medial aspects of the IC. All tissue stained for CO. Scale bar $=(\mathbf{A}) 75 \mu \mathrm{m}$, (B) 300 $\mu \mathrm{m},(\mathbf{C}) 600 \mu \mathrm{m}$.
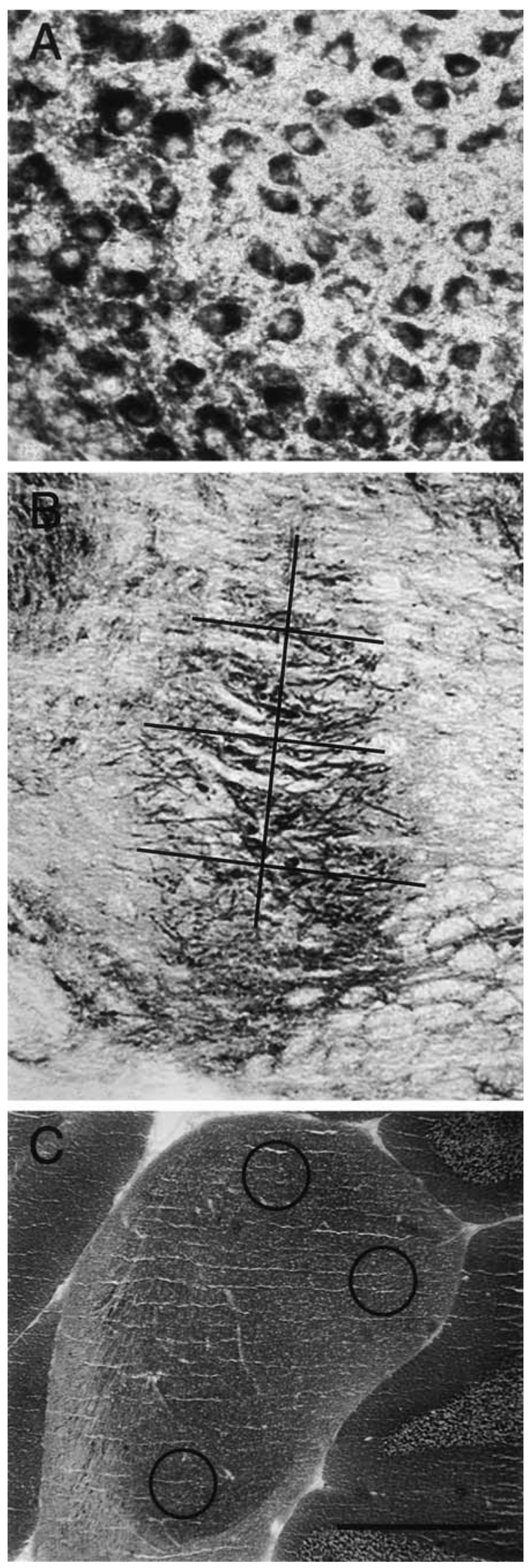
50 cells at approximately $20 \%$ of the anterior-toposterior dimension of the nucleus, at $40 \mathrm{X}$ magnification under bright-field illumination (Fig. 1A). Cell cytoplasm (nucleus excluded) was outlined and an OD measurement taken for each cell. Mean OD with standard deviation and standard errors, was determined for each AVCN.

2. MSO: (1) OD measurements were taken from three consecutive sections at approximately $50 \%$ of the anterior-to-posterior dimension of the MSO at 5X magnification. A line was drawn parallel to the cell bodies along the dorsal/ventral axis of the MSO, and OD measurements were taken for the COstained areas medial and lateral to this line (Fig. 1B). (2) In an effort to approximate the length of the medial and lateral MSO dendrites, the width of the CO-stained neuropil was measured at 10X magnification. A line perpendicular to the dorsal/ ventral axis of the nucleus was drawn from the medial to lateral edge of the neuropil (Fig. 1B). The apparent length of medial and lateral dendrites was estimated by measuring the length of this line medial and lateral to the dorsal/ventral axis. Measurements were taken from three consecutive sections located at approximately $40 \%$ of the anteriorto-posterior dimension of the nucleus, at the dorsal, middle, and ventral portions of the nucleus in each section. The mean neuropil width (in $\mu \mathrm{m}$ ) was calculated for each region.

3. LSO: OD measurements were taken from three consecutive sections at approximately $50 \%$ of the anterior-to-posterior dimension of the nucleus at $5 \mathrm{X}$ magnification. The nucleus was divided into medial and lateral portions (high- and low-frequency regions; Sanes and Siverls 1991), the area was outlined, and an OD measurement was obtained.

4. IC: OD measurements were taken at three locations within the IC-dorsal, ventral, and medialcorresponding to the low- and high-frequency portions of the central nucleus and an area outside the central nucleus, respectively (Fig. 1C). These measurements were made from three consecutive sections at 5X magnification and at a point approximately $70 \%$ of the anterior-to-posterior extent of the nucleus.

5. Control measurements: (i) For comparison to AVCN cell measurements, OD measurements were made of individual neurons in the motor nucleus of the trigeminal nerve. These were performed under the same magnification (40X) and identical microscope light and camera settings as the AVCN measurements. (2) For comparison to MSO, LSO, and IC nucleus measurements, whole nucleus OD measurements were taken from three consecutive sections of the abducens nucleus at the $50 \%$ point of the anterior-to-posterior extent. Measurements were taken at 5X magnification using identical microscope light and camera settings.

\section{Data analysis: CO optical density}

For each animal, the "corrected" OD measurements for MSO, LSO, and IC were obtained by dividing the averaged value from each nucleus on each side of the brain by the averaged value for the abducens nucleus on the same side. For AVCN, the mean neuron OD for each animal was divided by the mean OD for the trigeminal neurons on the same side of the brain. This correction allowed independent evaluation of ipsilateral and contralateral nuclei (e.g., comparison of OD in ipsilateral AVCN in experimental groups with the age- and survival-matched sham control groups). The abducens nucleus was chosen as the "control" area because of its well-defined borders and its location in the same sections as some of the auditory nuclei. While the abducens nucleus receives vestibular input, and thus theoretically may be affected by the manipulations, none of our animals demonstrated evidence of vestibular dysfunction after the experimental manipulations used. The trigeminal motor nucleus was chosen as a control for the AVCN neuron measurements for similar reasons. While Shore et al. (2000) identified projections from the trigeminal sensory nucleus to the cochlear nucleus, no projections from the motor neurons were noted. In order to insure that there was no effect of the experimental manipulation on either control nucleus, statistical analyses were carried out to look for significant left-right differences for both of these nuclei.

\section{Summary of Data Analysis:}

1. Raw data comparisons were not used for any statistical analyses. Because of differences in absolute CO density among animals, comparisons using raw data allow only for assessment of right to left differences within a single animal.

2. "Control" (abducens or trigeminal motor) nucleus correction: Correction to a neutral area of the brain allows for independent comparisons of each nucleus on each side of the brain between animals. In this way, it is possible to ascertain, for example, whether OD values after hearing loss go up in one nucleus

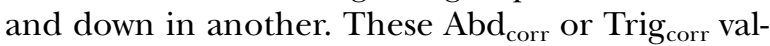
ues for experimental animals are compared with age- and survival-matched sham animals. Statistical analyses described below were performed on these transformed data.

While use of a sham control has the advantages described above, it will introduce variability into the analysis. As another method of data analysis, we also 
calculated the percent change between the two sides [ (contra - ipsi $) /$ contra] $\times 100$. These comparisons were made using the $\mathrm{Abd}_{\text {corr }}$ or Trig ${ }_{\text {corr }}$ data.

\section{Data analysis: MSO neuropil width}

For MSO width measurements, mean neuropil width in each region of the MSO (dorsal, middle, ventral) for each experimental group was compared with averaged sham measurements. As there were no statistically significant differences between sham groups (one-way ANOVA, $p>0.05$ ), all sham groups were combined for the purposes of statistical comparison with experimental groups. Mean neuropil width in the MSO as a whole (average of dorsal, middle, and ventral measurements) was also tabulated and compared with mean sham measurements.

In a second analysis, comparisons were made between length of "affected" vs. "unaffected" or control neuropil regions. MSO receives segregated inputs to the medial and lateral dendrites, with ipsilateral input to the lateral dendrites and contralateral input to the medial dendrites. Dendrites considered to be "affected" by a left ear hearing loss would be the left lateral and right medial dendrites. Control dendrites would be the right lateral and left medial dendrites. The widths of the neuropil containing affected and control dendrites were averaged and a percent change calculated by the following formula: [(Affected Control)/Control] $\times 100$.

\section{Statistical analysis}

\section{$\mathrm{CO}$ optical density measurements}

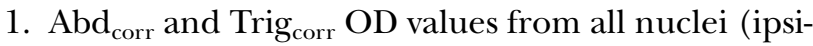
lateral and contralateral) were compared using a one-way ANOVA $(=0.05)$, grouping animals by age/survival manipulation groups. In the post hoc test (Fisher's PLSD), animals in each age/survival group were compared with their own sham controls.

2. Right to left differences were also compared using a one-way ANOVA $(=0.05)$.

3. In order to look for systematic differences between left and right brain measurements for the "control" areas (abducens and trigeminal motor nucleus), we compared left-to-right percent difference scores for each experimental group with that for sham ani- mals (grouped together) using Dunnett's test. No significant differences were observed, lending support to our supposition that these areas were unaffected by our experimental manipulation.

\section{MSO CO-stained neuropil measurements}

1. CO-stained neuropil in each of the three regions of the nucleus as well as the mean of all regions were compared for each experimental group and all combined sham animal data using a one-way ANOVA $(=0.05)$. Post hoc pairwise comparisons between groups were made using the Fisher PLSD test.

2. Comparisons between neuropil containing affected and control dendrites were also made using a paired $t$-test.

\section{Hearing threshold measurements}

Auditory brainstem evoked potential thresholds to both air- and bone-conducted stimuli were obtained in a separate group of animals following malleus removal and cochlear ablation. The methodology and results of the measurements were discussed in detail in a previous publication (Tucci et al. 1999) and demonstrate that the hearing loss produced by malleus removal is purely a CHL (no associated SNHL), ranging from 35 to $55 \mathrm{~dB}$ threshold shift across frequency, compared with normal hearing animals. No hearing was detected in the affected ear following CA.

\section{RESULTS}

\section{Optical density measurements of $\mathrm{CO}$ activity}

Qualitative results. Effects of unilateral surgical manipulations were most apparent in the second-order neurons of the AVCN. (This is the only nucleus for which optical density measurements of individual neurons were made.) Figure 2 shows representative right and left (right and left of figure) coronal sections through the rostral AVCN of sham $(\mathrm{A}, \mathrm{B})$, left ear cochlear ablation (C,D), and left ear conductive hearing loss (E,F) animals. While sham animals show no discernible right-left differences, the difference between sides for the cochlear ablation animals is readily apparent. Optical density of neurons in the right AVCN, which receives normal input from the
FIG. 2. Coronal sections through the rostral AVCN showing cytochrome oxidase density in (A,B) left ear sham (C,D) cochlear ablation and $(\mathbf{E}, \mathbf{F})$ conductive hearing loss animals (all from "adult" groups, left and right sides from same animal in each case). In all cases, the nucleus ipsilateral to the manipulated ear is shown on the left and the unmanipulated side is shown on the right. Scale bar $=50 \mu \mathrm{m}$. 

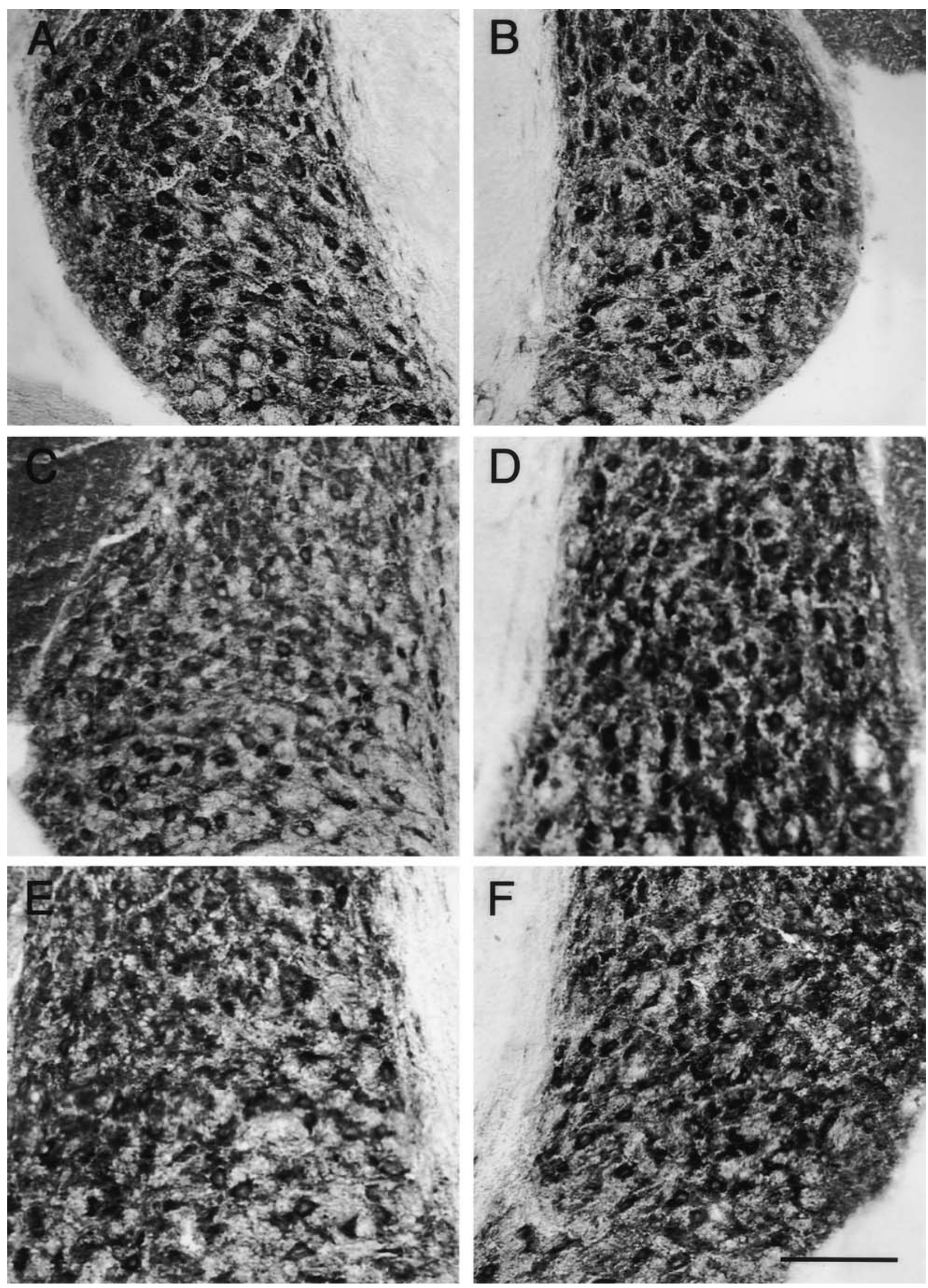
right cochlear nerve, is greater than that in the left AVCN, which receives afferent input from the ablated ear. While less obvious, there are also notable differences following left ear conductive hearing loss (bottom panel), where $\mathrm{CO}$ staining is greater in the right AVCN.

Quantitative results. Results of our quantitative measurements of OD are shown in Figures 3 and 4. As described above, for each age and survival time, we measured $\mathrm{CO}$ optical density in experimental animals as well as sham animals. Measurements in each nucleus were corrected against a neutral region of the brain to allow for absolute differences in staining between animals. These abducens-or trigeminal-corrected values then were used for statistical comparisons (oneway ANOVA) to determine where significant changes in OD occur. For each nucleus, post hoc comparisons (Fisher PLSD) were made between experimental animals and the appropriate sham group. However, for simplicity in presenting the data, we have calculated for each region the percent change in OD for experimental compared with sham animals. These data are plotted in Figures 3 and 4. For bars above the $0 \%$ change line, experimental animal OD measurements were greater than that for sham; for bars below the line, experimental animal OD measurements were less than that for sham. OD values used for experimental and sham animal measurements were Trig corr $_{\text {for }}$ AVCN cell measurements and $\mathrm{Abd}_{\text {corr }}$ for MSO, LSO, and IC measurements.

Figure 3 shows data for animals that underwent a left ear cochlear ablation either at P21 or as an adult (age 6-8 weeks). Two days after left CA, there were no significant changes in $\mathrm{CO}$ optical density, with the exception of an increase in OD in the right IC (Fig. 3A). However, three weeks after CA (Fig. 3B), significant $(p<0.05)$ decreases in $\mathrm{CO}$ optical density were observed in the left AVCN, left lateral MSO, and right medial MSO. In the adult animals (Fig. 3C), changes in $\mathrm{CO}$ optical density were more widespread, with significant decreases $(p<0.05)$ observed in left AVCN, left lateral MSO, right and left medial MSO, and lateral LSO on both the right and left sides of the brain. Optical density also decreased slightly in the other auditory nuclei, although these decreases failed to reach statistical significance.

Figure 4 shows OD data for animals that underwent a left ear conductive hearing loss at P21 or as an adult. Data are clustered at the 0\% change line for P21 animals at both the 2 day and 3 week survival times, indicating no CO changes with CHL (Fig. 4A and B). Significant $(p<0.05)$ changes were observed only for the adult animals (Fig. 4C), and were seen in both the left and right AVCN and left lateral LSO. A left ear conductive hearing loss was associated with a decrease in $\mathrm{CO}$ density in the left AVCN and an increase in CO density in the right AVCN, contralateral to the CHL.

Left-right differences were also evaluated using percent difference scores $\{[($ right - left $) /$ right $] \times$ $100\}$ rather than sham-corrected comparisons. While sham-corrected comparisons are valuable because they allow statistically reliable comparisons between animals, more subtle differences within an individual animal may be obscured because of the variability introduced by use of the sham animals. Using an ANOVA, statistically significant right-left differences were found only for the AVCN. Therefore, no significant differences above those detected using sham-corrected comparisons were identified.

\section{MSO neuropil measurements}

Results of width measurements of CO-stained neuropil for animals with left ear CA and CHL are shown in Figures 5 and 6, respectively. Bar graphs show mean neuropil width (in $\mathrm{mm}$ ) for both experimental and sham control animals. Asterisks indicate statistically significant differences (one-way ANOVA and Fisher post hoc pairwise comparisons).

It is apparent that there is a tendency in P21 animals for MSO CO-stained neuropil in both the medial and lateral fields on both sides of the brain to shorten following unilateral CA (Figs. 5A and B). However, these changes did not reach statistical significance. In the adult animals (Fig. 5C), there was a tendency for the length of CO-stained neuropil to increase compared with sham animals. However, these changes also failed to reach statistical significance.

Effects of unilateral CHL on the width of COstained neuropil is shown in Figure 6. Findings for the P21 animals surviving 2 days are similar to the results of CA (Fig. 6A). However, in the P21 animals surviving 3 weeks, we observed marked increases in CO-stained neuropil width throughout the MSO, in both medial and lateral fields, on both sides of the brain (Fig. 6B). All differences were statistically significant $(p<0.05)$. Findings for the adult group were similar to findings for the CA experimental group, with no significant differences observed. Although not significantly different from sham, effects were of similar magnitude and direction for both medial and lateral neuropil regions, for both the right and left sides of the brain for all animal groups.

Results of a second analysis of MSO neuropil width measurements are shown in Figure 7. This analysis is based on comparison of dendritic fields that receive input from the ear with the hearing loss vs. the normalhearing ear. For this analysis, percent change in neuropil width (which may reflect changes in dendritic length) was calculated using the following formula: 


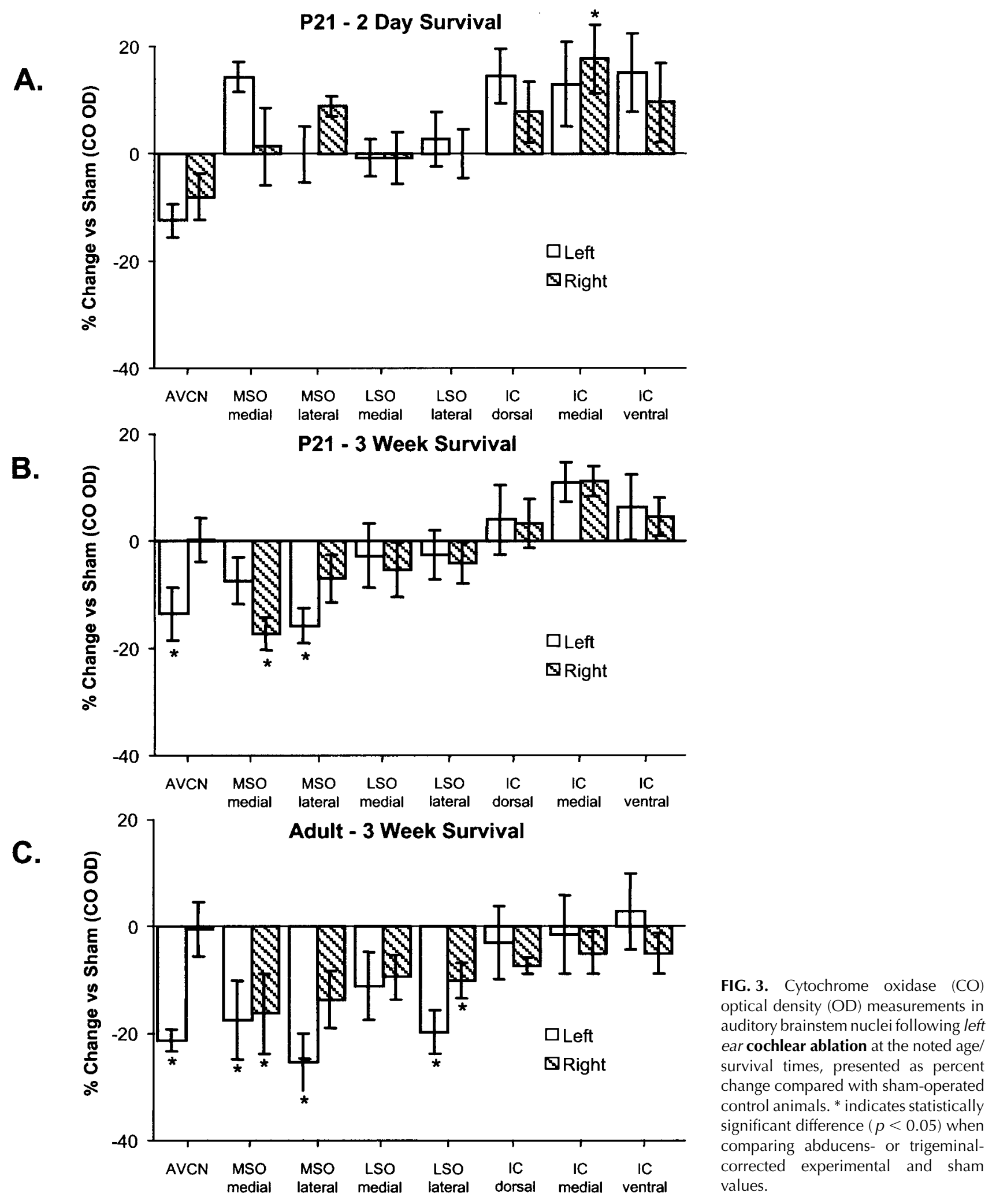

[ (affected - control) / control] $\times 100$; "affected" dendrites are those that receive input from the left cochlear nucleus (CN, i.e., left lateral and right medial dendrites) and "control," or unaffected dendrites, are those that receive input from the right $\mathrm{CN}$ (i.e., left medial and right lateral dendrites). These measures are not sham-corrected. The analysis was performed separately for dorsal, middle, and ventral neuropil 

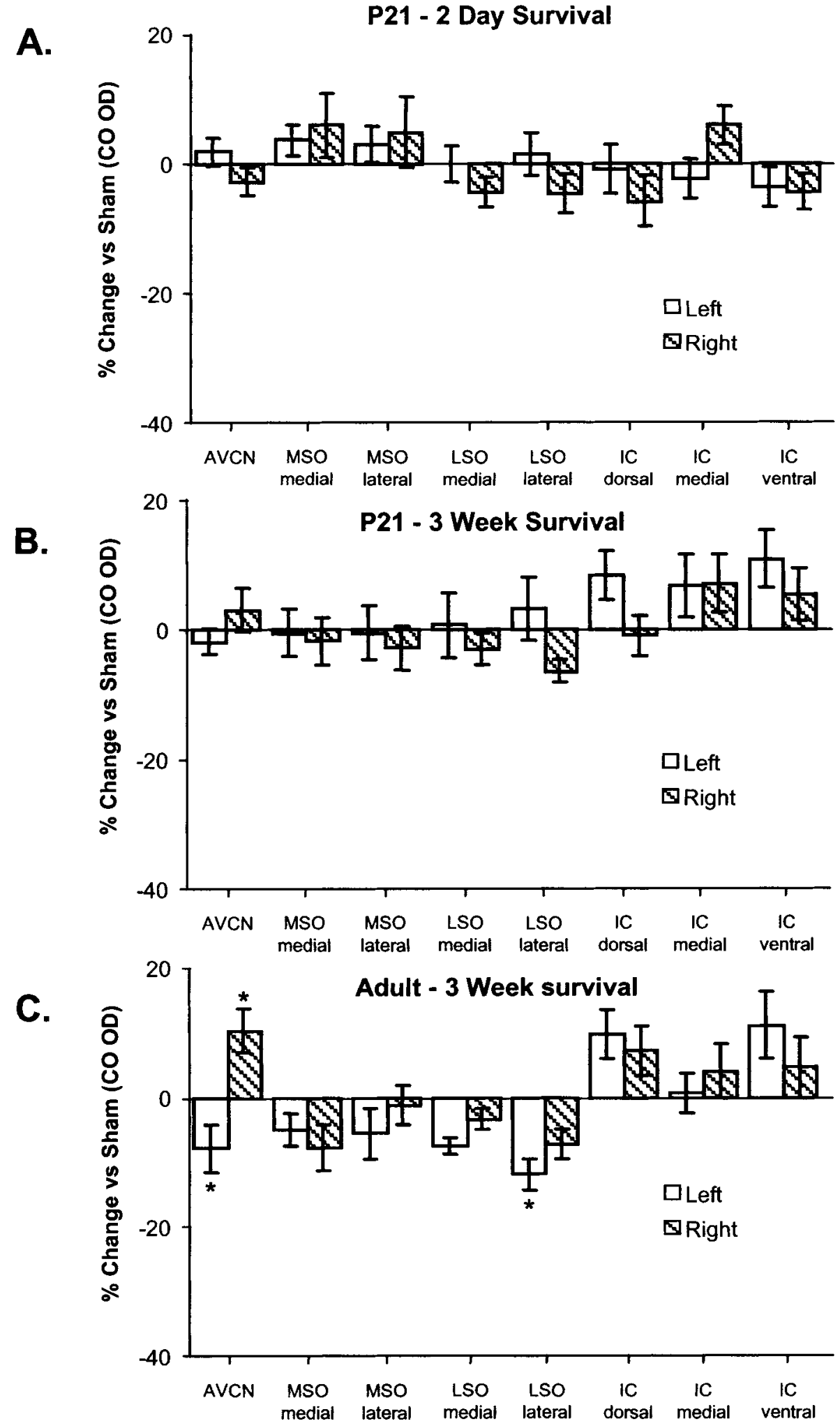

FIG. 4. Cytochrome oxidase (CO) optical density (OD) measurements in auditory brainstem nuclei following left ear malleus removal at the noted age/ survival times, presented as percent change compared with sham-operated control animals. * indicates statistically significant comparison $(p<0.05)$ for experimental vs. sham.

regions to look for any frequency-specific changes. (MSO is tonotopically organized, with low frequencies most dorsal.) As shown in Figure 7, results were very similar for all regions of MSO. "Affected" neuropil regions were significantly shorter than control regions in both the P21 and adult animals following CA. This change was in the approximate range of $15 \%-25 \%$. Following CHL in the adult animals, there was a $10 \%-$ 

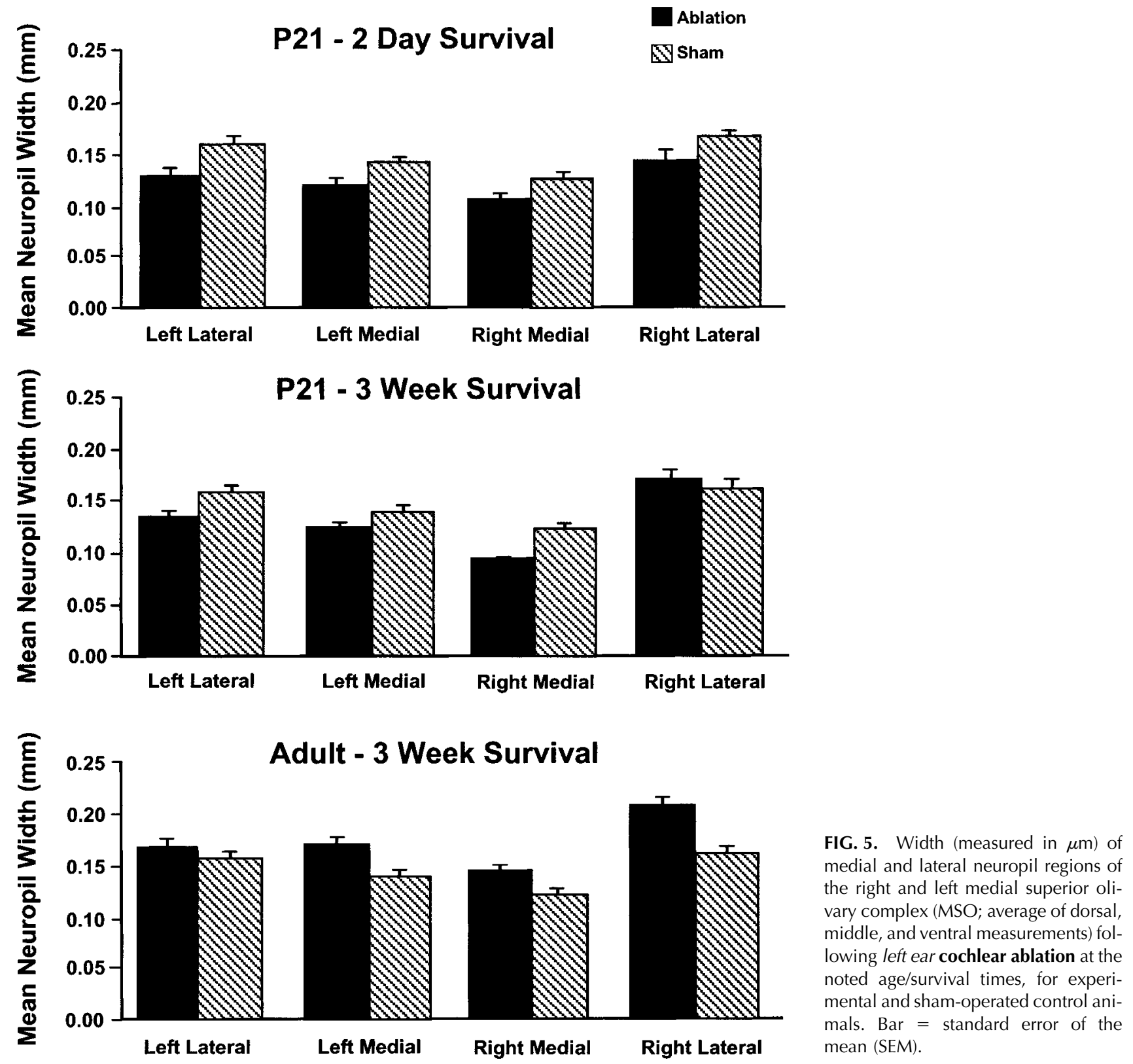

$15 \%$ change in the affected dendrites. Although this was slightly greater than that for sham animals or P21 CHL animals, this change failed to achieve statistical significance.

\section{DISCUSSION}

The major finding of this study was that unilateral conductive hearing loss is associated with significant metabolic, and possibly structural, changes in the central auditory system. Levels of oxidative enzymes in neuronal tissue are thought to be tightly coupled to levels of electrical activity (Lowry 1975; Wong-Riley et al. 1978; Mawe and Gershon 1986, Wong-Riley et al. 1981). Unilateral CHL in adult animals resulted in a significant decrease in $\mathrm{CO}$ density in second-order neurons of the left (ipsilateral) AVCN; there was also a significant increase in CO density in the right (contralateral) AVCN. There were no significant changes in CO density identified in animals that underwent CHL at P21. CO optical density measured with this histochemical stain has been shown to relate to $\mathrm{CO}$ activity measured biochemically (Darriet et al. 1986), suggesting the density changes we measure reflect changes in $\mathrm{CO}$ activity.

In this study, we used CO staining as an index of length of dendrites in the MSO. This measure may or 

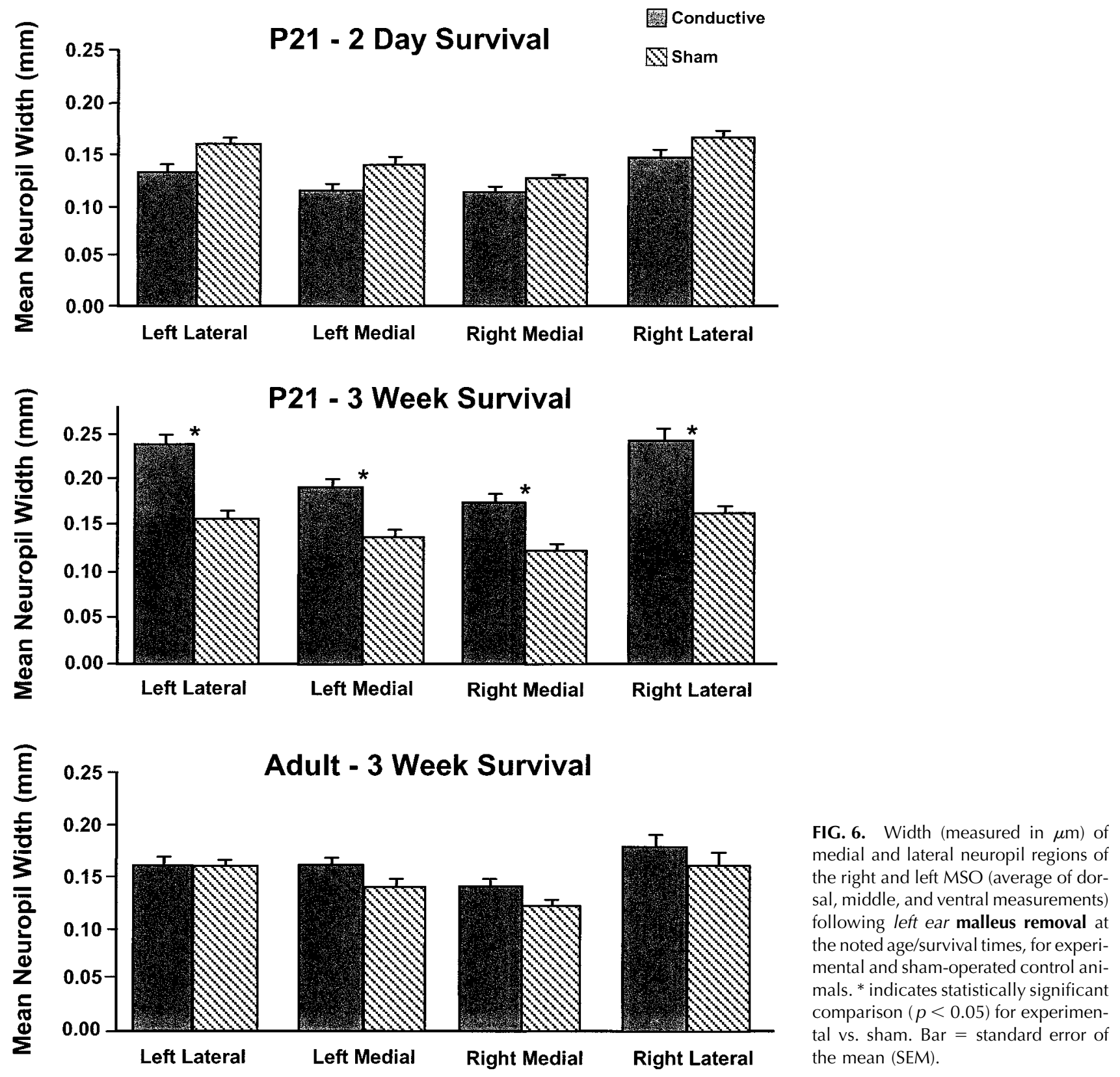

may not be reflective of the actual dendritic length. It is known that $\mathrm{CO}$ distribution within single neurons is nonhomogeneous, and differences in $\mathrm{CO}$ levels are often apparent not only between the cell body and its processes, but also between segments of the same dendritic tree (Wong-Riley 1989). Accurate measures of dendritic length would require use of more direct staining techniques, such as Golgi staining. Hence, we will refer to our measure as "apparent dendritic length."

Previous investigations have shown that unilateral CHL results in shortening of the lateral ipsilateral and medial contralateral dendrites that receive their primary afferent input from the manipulated ear (Feng and Rogowski 1980; Conlee and Parks 1981, 1983; Gray et al. 1982; Smith et al. 1983). In this study, we demonstrated no differential effect of CHL on these segregated dendritic fields. An unexpected finding was that MSO apparent dendritic length increased following CHL in the P21 animals that survived 3 weeks. As no abnormalities in $\mathrm{CO}$ density were identified in these younger animals, this increase may reflect a capacity for compensatory change in this age group, which is still undergoing active CAS development. Taken together, these findings may indicate that the younger animals are more sensitive to 
A.
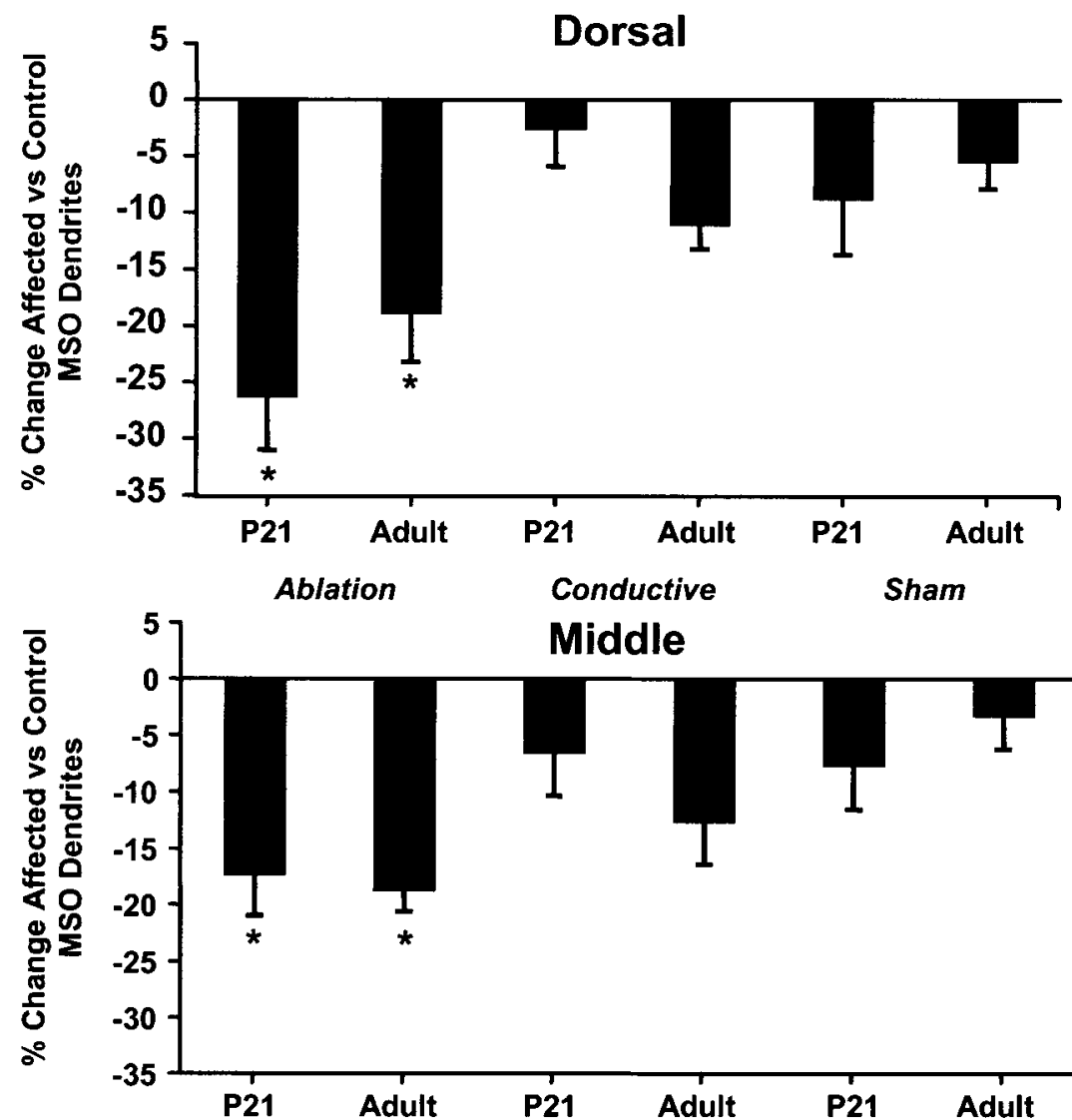

Ablation
B.

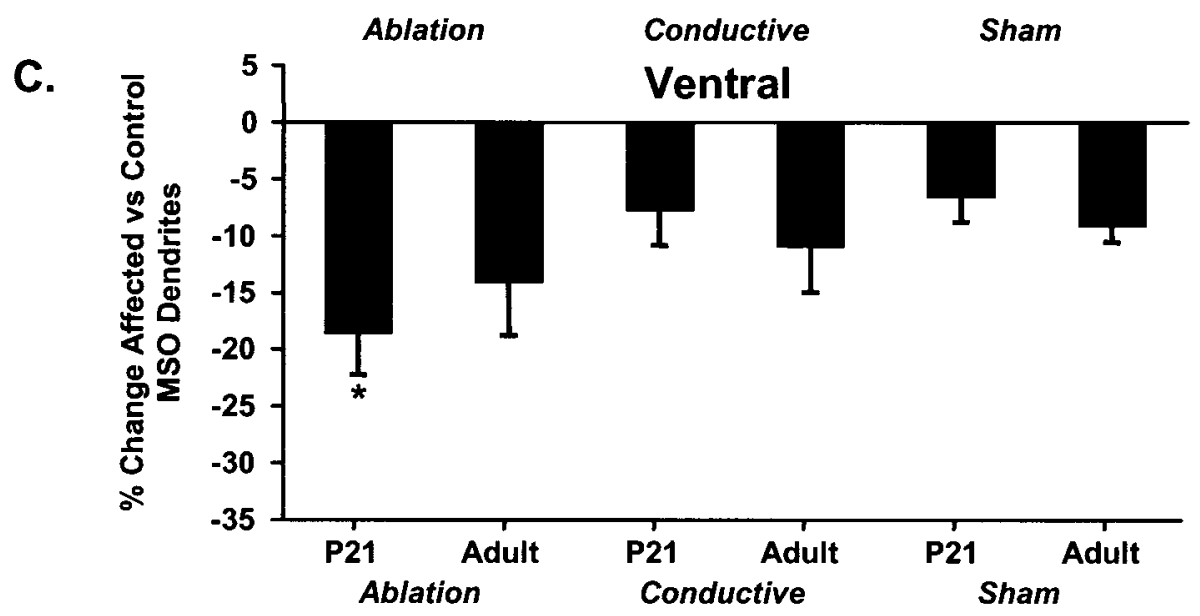

FIG. 7. Percentage change in width of neuropil ("apparent" length of dendrites that receive input from the experimental ear vs. those that receive input from the nonmanipulated ear) computed as [(Affected - Control)/Control] $\times 100$. Survival time for P21 animals $=3$ weeks. ${ }^{*}$ indicates statistically significant difference compared with sham animals $(p<0.05)$. Bar $=$ SEM. changes in afferent input (evidenced by greater changes in neuronal activity, or 2-DG uptake) but that their central auditory systems are also more plastic, i.e., capable of responding with structural and functional changes to minimize the negative impact of the hearing deficit. At least, our findings demonstrate that immature and adult animals respond differently to loss of afferent input.

As expected (Wong-Riley et al. 1978; Hyde and Durham 1990), unilateral CA was associated with significant decreases in $\mathrm{CO}$ density, particularly in $\mathrm{CN}$ and SOC. As with CHL, effects on CO density were greatest in the adult animals. Also as expected (Rubel et al. 1981), we demonstrated changes in apparent length of MSO dendrites that receive input from the ablated ear. Unilateral CA had no effect on MSO apparent dendrite length when compared with sham controls. However, when affected and unaffected dendrites were compared directly, there was an apparent $15 \%-25 \%$ decrease in length of affected dendrites (those that receive afferent input from the ablated ear) compared with control dendrites. 
Comparison of the 2-DG and CO methods and 2-DG findings following unilateral $\mathrm{CHL}$

Both cytochrome oxidase histochemistry and 2-deoxyglucose autoradiography are thought to be positively related to levels of neuronal activity. Whereas 2-DG uptake reflects neuronal glucose consumption, which may be expected to fluctuate on a minute-to-minute basis, $\mathrm{CO}$ histochemistry reflects the metabolic capacity of the cell, which is regulated over hours to days. A cell may not utilize glucose at the moment 2-DG levels are examined but retains the capacity to respond to increased levels of afferent input or oxidative demand and contains high levels of CO. Hence, the two techniques reflect different information and certainly may show different patterns of response to changes in afferent input.

In a previous experiment (Tucci et al. 1999), we found that either unilateral CHL or CA resulted in a marked decrease in 2-DG uptake in the nuclei that receive major afferent input from the manipulated ear. Although significant decreases in glucose utilization were observed in both young and adult animals, decreases were greatest, and most similar for CHL and $\mathrm{CA}$, in the young (P21) animals. The current study was performed using the same animals as used for the 2-DG study. Metabolic capacity evaluated with $\mathrm{CO}$ staining was much less dramatically altered by hearing loss, and, unlike 2-DG uptake, apparent CO activity changes following both CHL and CA were seen primarily in adult animals. It is clear from a comparison of these results that changes seen in oxidative metabolic capacity do not mirror minute-to-minute fluctuations in glucose consumption. It is possible that an increase in oxidative capacity is observed in the adult animals and not in the young animals because absolute baseline metabolic levels are greater in the young animals and, thus, there is less need to increase levels of CO. However, we could not measure $\mathrm{CO}$ activity quantitatively in our material. Alternatively, the adult animals may have a greater capacity to up-regulate metabolism in response to changes in afferent input. A third possibility is that changes in cell size, which were not assessed in the current study, could affect the apparent CO staining density, potentially masking a reduction in $\mathrm{CO}$ activity in the younger animals. For example, if neurons in the younger animals decrease in area more than in older animals in response to the experimental manipulation, this may result in an apparent increase in CO staining per cell area. We are currently assessing cell size changes following CHL as part of another study.

\section{Changes in $\mathrm{CO}$ with deafferentation}

Previous studies of oxidative enzyme activity in the CAS following deafferentation have shown significant decreases in $\mathrm{CO}$ and in two Krebs cycle enzymessuccinate dehydrogenase and malate dehydrogenase-following unilateral cochlear removal in the chick (Durham and Rubel 1985; Hyde and Durham 1990; Durham et al. 1993). Subsequent studies by Hyde and Durham (1993a, b) demonstrated that changes in CO density reflect rapid changes in mitochondria in the deafferented neurons. They observed an initial proliferation of mitochondria in all neurons which was associated with a short-term increase in oxidative enzyme activity; neurons destined to die as a result of deafferentation were observed to develop ultrastructural abnormalities, including smaller mitochondria which stained poorly for $\mathrm{CO}$. The early increases in CO staining were observed in young (10 days) but not in older (56 week) chickens (Hyde and Durham 1990), reflecting age-specific results of deafferentation that have also been demonstrated in other studies (Rubel et al. 1990; Tierney et al. 1997). Although the time course of CO change was not a focus of this study, in the P21 2-day survival animals only we observed an overall increase in $\mathrm{CO}$ density in many nuclei central to the AVCN. However, only the increase in one (the medial) portion of the IC reached statistical significance.

Changes in postsynaptic neural structure and function are known to occur in response to a manipulation of afferent input during a critical period of development. The exact relationship between the critical period and milestones in auditory development varies among species and is specific to the structural or functional measure employed for assessment. Tierney et al. (1997) have shown that gerbils are susceptible to deafferentation-induced cell death only until 7-9 days after birth, several days prior to the onset of hearing at P12 (Woolf and Ryan 1984, 1985). Gerbils at P21 are still undergoing developmental change, as auditory function is not fully mature in the gerbil until at least P30 (Woolf and Ryan 1984). Studies by Hashisaki and Rubel (1989) and Sie and Rubel (1992) in the gerbil have shown that deafferentation by cochlea removal or the chronic application of tetrodotoxin (TTX) results in changes in the size of large spherical cells in AVCN and in protein synthesis in AVCN neurons. These changes occur well past the critical period for cell death. Our P21 animals were not expected to experience neuronal death following cochlea removal. Therefore, we would not expect to see the same time course of changes in $\mathrm{CO}$ density as in the Hyde and Durham (1994a, b) studies, which have been shown to reflect changes associated with cell death.

Cytochrome oxidase: effects of unilateral $\mathrm{CHL}$

We found that $\mathrm{CO}$ density was decreased in the left, or ipsilateral, AVCN and increased in the right, or contra- 
lateral, AVCN after CHL. The effect on the right AVCN is larger than the opposite effect observed in the left AVCN. Two lines of evidence in the literature support the finding of changes on the nonmanipulated side of the brain.

Coleman and O'Connor (1979) reported mean cell areas of large spherical cells in AVCN of the rat following unilateral CHL and in control animals. While most studies that have examined the effects of unilateral CHL on CAS anatomy have measured only left-right differences, these authors measured the two sides independently, allowing for the detection of any changes on the nonmanipulated side of the brain. They showed a 17\% decrease in cell area ipsilateral to the conductive loss. They also showed a slight $(\sim 5 \%)$ increase in AVCN cell area on the contralateral side.

Changes in the afferent projection from the $\mathrm{CN}$ to the IC have been reported following both unilateral CA (Nordeen et al. 1983b; Moore and Kitzes 1985) and CHL (Moore et al. 1989). As detailed by Nordeen and colleagues (1983a), the IC of the gerbil receives a major projection from the contralateral CN. A smaller, ipsilateral projection from the $\mathrm{CN}$ to $\mathrm{IC}$ originates from all three divisions of the $\mathrm{CN}$ and is thought to be biased toward low frequencies. Under normal circumstances, the projections from the AVCN and PVCN are sparse and those from the DCN are more substantial (approximately one-third as large as the contralateral projection). Unilateral cochlear destruction results in a significant increase in the size of the ipsilateral CN-IC projection on the opposite side of the brain (Nordeen et al. 1983b). Anatomical changes were accompanied by increased ipsilaterally evoked excitatory activity. This structural and functional alteration of a CAS pathway appears to be due to the presence of unequal activity from the two ears and not deafferentation per se, as no changes in the $\mathrm{CN}-\mathrm{IC}$ pathway were observed following bilateral cochlear ablation (Moore 1990). Moore et al. (1989) identified similar changes in the ipsilateral $\mathrm{CN}-\mathrm{IC}$ projection following unilateral CHL in the neonatal ferret. All divisions of the $\mathrm{CN}$ were found to contribute to the increase in the ipsilateral projection. Thus, upregulation of $\mathrm{CN}$ neurons may occur secondary to $\mathrm{CA}$ or CHL in the contralateral ear. This could account for the increase in CO uptake in the right AVCN found in the present study.

Two findings in the present study do not support this interpretation. First, in the studies cited above, changes were identified in the ipsilateral $\mathrm{CN}-\mathrm{IC}$ projection following CA or CHL in neonatal but not in adult animals. However, we found no changes in right AVCN CO density in animals that were subjected to hearing loss at P21; changes were seen only in adult animals. Further, no increase in AVCN CO density was observed after $\mathrm{CA}$, with the exception of the P21 short survival group, and this change did not achieve statistical significance.

\section{Effects of unilateral CA on apparent dendritic length}

MSO dendritic fields that receive primary afferent input from the ablated ear were apparently shorter (based on the width of CO-stained neuropil) than those that receive input from the normal-hearing ear. Similar results were reported by Rubel et al. (1981). In their study, unilateral cochlea removal in the 10-15day-old chick resulted in a slight reduction in the length of the ipsilateral dorsal and contralateral ventral dendritic arbors in $\mathrm{n}$. laminaris (NL; the avian equivalent of MSO). These dendritic fields receive input from the deafferented ear. Compared with transection of the crossed dorsal cochlear tract, which completely deafferents the ventral dendrites of NL bilaterally, the effects of cochlear removal are modest (Rubel et al. 1981; Deitch and Rubel 1984). Although the quantitative data needed to make a comparison are not available, it is interesting to note that the effects of cochlear removal on dendrites of MSO or NL may be more modest than the effects of unilateral CHL.

\section{Effects of unilateral CHL on apparent dendritic length}

The effects of unilateral and bilateral CHL on MSO dendrites have been noted previously. Feng and Rogowski (1980) examined dendritic length of MSO cells in rats following either unilateral or bilateral CHL at day P12 and 60 days after birth. The extent of dendritic branching of the neurons in MSO was measured and classified as "right dominant,' "left dominant," or "equal dominance." Control and bilateral CHL animals exhibited equal numbers of right and left dominant neurons. However, monaurally occluded animals were demonstrated to have a higher proportion of dendrites that were more developed on the side innervated by the normal ear. Since they did not compare values with sham animals, it is not known if deprived dendrites were shorter than normal or the nondeprived dendrites were longer, or both.

Gray et al. (1982) and Conlee and Parks (1983) described similar findings in the chick auditory system following unilateral earplug placement at embryonic day 18-19. Deprived dendrites in experimental animals were significantly shorter than those same dendrites in control animals. Nondeprived dendrites were also similar to control animals, so no compensatory changes were identified (Conlee and Parks 1983). Smith et al. (1983) used the known tonotopic organization of $\mathrm{n}$. laminaris, the avian equivalent of MSO, to investigate frequency-specific effects of unilateral CHL 
in the chick. They identified a differential effect across frequency for animals that underwent placement of a unilateral earplug during the late embryonic stage. Shortening of the affected dendrites occurred for high-frequency fibers, while low-frequency fibers actually increased in length. One hypothesis suggested by the authors to explain this result is that mass loading of the tympanic membrane by the earplug may have actually increased low-frequency transmission through the middle ear. Such an effect would not be expected with malleus removal, as in the present study, and no frequency-specific effects were noted, based on measurements of dendritic length in the dorsal vs. ventral aspects of MSO (low- vs. high-frequency regions). An alternative explanation of the findings by Smith et al. (1983) could be that the earplug procedure used caused damage to the high-frequency region of the cochlea, with a resultant decrease in dendritic length as seen with cochlea removal. The finding of increased dendritic length in the low-frequency area of $\mathrm{n}$. laminaris is consistent with the results of the present study.

It is difficult to explain why CHL might result in an increase rather than a decrease (as in CA) in the length of the MSO dendrites. It should be noted that the apparent increase in length could also reflect an increase in $\mathrm{CO}$ density in the more distal portions of the dendrites; our method of analysis would not differentiate between these two possibilities. Further, it is not at all clear why both the affected and (presumably) unaffected dendrites are influenced by this manipulation in afferent input. It is possible that the dendrites that have not been deafferented, as with CA, are capable of regulating length and function in an attempt to increase input to the MSO neurons. The increase in length of the unaffected dendrites may reflect a compensatory mechanism. As with the change in ratio of ipsilateral and contralateral $\mathrm{CN}$ input to the inferior colliculus, a competitive interaction may influence the size of the MSO dendrites. The role of afferent competition in development of binocular vision has been well demonstrated (Sherman and Spear 1982; Friedlander et al. 1991), and a similar mechanism may be important in the development of innervation and maintenance of normal function in auditory nuclei that receive bilateral input (Nordeen et al. 1983b).

\section{CHL and afferent activity}

CHL is associated with changes in afferent activity, which lead to structural and functional CAS changes. While many of these changes mimic, to a lesser degree, those seen with deafferentation, some may be fundamentally different, particularly in binaurally innervated nuclei such as the MSO. This nucleus appears to be more affected by changes in the balance of input between the two ears than the absolute level of input. This makes sense, since absolute levels of stimulation may vary considerably under normal conditions.

CHL resulted in a clear decrease in oxidative metabolism, as measured by $\mathrm{CO}$ density, in the ipsilateral AVCN. In light of the evidence that these neurons are regulated by afferent input, an explanation must invoke a relationship between these anatomical and functional changes and changes in neuronal activity. It is possible that CHL does result in a long-term change in spontaneous auditory nerve activity. It is possible that CHL does result in a long-term change in spontaneous auditory nerve activity. Although investigation in the chick indicates that activity in secondorder neurons is unchanged following columella removal (Tucci et al. 1987), auditory nerve activity following CHL in a mammal has never been measured. Second, it is possible that the overall level of activity, including spontaneous as well as sound-evoked activity, is important in regulation of CAS structure and function. There is some evidence that normal environmental sounds, including variations in patterns of auditory stimulation, are necessary for normal development of the CAS (Sanes and Constantine-Paton 1985a, b; Keilmann and Herdegen 1997; Ryugo et al. 1998).

\section{ACKNOWLEDGMENTS}

The authors wish to thank Dr. Deb Park for valuable technical assistance and assistance with statistical analysis, and Sandy Parsons for expert technical assistance. We gratefully acknowledge the helpful comments of two anonymous reviewers. Supported by NIH grants K08DC00125 (DLT), DC01589 (DD), and DC00135 (NBC).

\section{REFERENCES}

Blatchley BJ, Williams JE, Coleman JR. Age-dependent effect of acoustic deprivation on spherical cells of the rat anteroventral cochlear nucleus. Exp. Neurol. 80:81-93, 1983.

Born DE, Durham D, RubEL EW. Afferent influences on brainstem auditory nuclei in the chick: Nucleus magnocellularis neuronal activity following cochlea removal. Brain Res. 557:37-47, 1991.

BORN DE, RUBEL EW. Afferent influences on brain stem auditory nuclei of the chicken: Neuron number and size following cochlea removal. J. Comp. Neurol. 231:435-445, 1985.

BORN DE, RUBEL EW. Afferent influences on brain stem auditory nuclei of the chicken: presynaptic action potentials regulate protein synthesis in nucleus magnocellularis neurons. J. Neurosci. 8:901-919, 1988.

Brugge JF, Orman SS, Coleman JR, Chan JCK, Phillips DP. Binaural interactions in cortical area $\mathrm{AI}$ of cats reared with unilateral atresia of the external ear canal. Hear. Res. 20:275-287, 1985.

CLOPTON BM, Silverman MS. Plasticity of binaural interaction. II. Critical period and changes in midline response. J. Neurophysiol. 40:1275-1280, 1977.

Clopton BM, Silverman MS. Changes in latency and duration of 
neural responding following developmental auditory deprivation. Exp. Brain Res. 32:39-47, 1978.

Coleman JR, BLatchley BJ, Williams JE. Development of the dorsal and ventral cochlear nuclei in rat and effects of acoustic deprivation. Dev. Brain Res. 4:119-123, 1982.

COLEMAN JR, O'CONNOR P. Effects of monaural and binaural sound deprivation on cell development in the anteroventral cochlear nucleus of rats. Exp. Neurol. 64:553-566, 1979.

CONLEE JW, PARKS TN. Age- and position-dependent effects of monaural acoustic deprivation in nucleus magnocellularis of the chicken. J. Comp. Neurol 202:373-384, 1981.

CONLEE JW, PARKS TN. Late appearance and deprivation-sensitive growth of permanent dendrites in the avian cochlear nucleus, $n$. magnocellularis. J. Comp. Neurol. 217:216-226, 1983.

DARRIET D, Der T, Collins RC. Distribution of cytochrome oxidase in rat brain: studies with diaminobenzidine histochemistry in vitro and $[14 \mathrm{C}]$ cyanide tissue labeling in vivo. J. Cereb. Blood Flow Metab. 6:8-14, 1986.

DeItch JS, RUBEL EW. Afferent influences on brain stem auditory nuclei of the chicken: Time course and specificity of dendritic atrophy following deafferentation. J. Comp. Neurol. 229:66-79, 1984.

DOYLE WJ, WeBsTER DB. Neonatal conductive hearing loss does not compromise brainstem auditory function and structure in rhesus monkeys. Hear. Res. 54:145-151, 1991.

Durham D, MATSChInSky FMM, Rubel EW. Altered malate dehydrogenase activity in $\mathrm{n}$. magnocellularis of the chicken following cochlea removal. Hear. Res. 70:151-159, 1993.

Durham D, Rubel EW. Afferent influences on brain stem auditory nuclei of the chicken: Changes in succinate dehydrogenase activity following cochlea removal. J. Comp. Neurol. 231:446-456, 1985.

FENG AS, RogOwski BA. Effects of monaural and binaural occlusion on the morphology of neurons in the medial superior olivary nucleus of the rat. Brain Res. 189:530-534, 1980.

Friedlander MJ, Martin KAC, WASSENHOVE-MCCARTHY D. Effects of monocular visual deprivation on geniculocortical innervation of area 18 in cat. J. Neurosci. 11:3268-3288, 1991.

Gray L, Smith Z, Rubel EW. Developmental and experimental changes in dendritic symmetry in $n$. laminaris of the chick. Brain Res. 244:360-364, 1982.

HALl JW, DERLACKI EL. Binaural hearing after middle ear surgery: masking-level differences for interaural time and amplitude cues. Audiology 27:89-98, 1988.

Hall JW, Grose JH, Pillsbury HC. Long-term effects of chronic otitis media on binaural hearing in children. Arch. Otolaryngol. Head Neck Surg. 121:847-852, 1995.

HASHISAKI GT, RUBEL EW. Effects of unilateral cochlea removal on anteroventral cochlear nucleus neurons in developing gerbils. J. Comp. Neurol. 283:465-473, 1989.

Hyde GE, Durham D. Cytochrome oxidase response to cochlea removal in chicken auditory brainstem neurons. J. Comp. Neurol. 297:329-339, 1990.

Hyde GE, Durham D. Rapid increases in mitochondrial volume in nucleus magnocellularis neurons following cochlea removal. J. Comp. Neurol. 339:27-48, 1994a.

Hyde GE, DuRham D. Increased deafferentation-induced cell death in chick brainstem auditory neurons following blockade of mitochondrial protein synthesis with chloramphenicol. J. Neurosci. 14:291-300, 1994b.

HYSON RL, RUBEL EW. Transneuronal regulation of protein synthesis in the brain-stem auditory system of the chick requires synaptic activation. J. Neurosci. 9:2835-2845, 1989.

Keilmann A, Herdegen T. The c-Fos transcription factor in the auditory pathway of the juvenile rat: effects of acoustic deprivation and repetitive stimulation. Brain Res. 753:291-298, 1997.

KNUDSEN EI. Mechanisms of experienced-dependent plasticity in the auditory localization pathway of the barn owl. J. Comp. Physiol. 185:305-321, 1999.

Koerber KC, Pfeiffer RR, Warr WB, Kiang YS. Spontaneous spike discharges from single units in the cochlear nucleus after destruction of the cochlea. Exp. Neurol. 16:119-130, 1966.

LOWRY OH. Energy metabolism in brain and its control. In: Ingvar DH, Lassen NA, (eds) Brain Work. The Coupling of Function, Metabolism and Blood Flow in the Brain. Academic Press New York, 1975 , p. $48-63$.

MAWE GM, GERSHON MD. Functional heterogeneity in the myenteric plexus: Demonstration using cytochrome oxidase as a verified cytochemical probe of the activity of individual enteric neurons. J. Comp. Neurol. 249:381-391, 1986.

MOORE DR. Auditory brainstem of the ferret: Bilateral cochlear lesions in infancy do not affect the number of neurons projecting from the cochlear nucleus to the inferior colliculus. Exp. Brain Res. 54:125-130, 1990.

MOORE DR. Development and plasticity of the ferret auditory system. In: Altschuler RA, Bobbin RP, Clopton BM, Hoffman DW, (eds) Neurobiology of Hearing: The Central Auditory System. Raven Press New York, 1991, p. 461-475.

Moore DR, Hutchings ME, King AJ, Kowalchuk NE. Auditory brainstem of the ferret: Some effects of rearing with a unilateral ear plug on the cochlea, cochlear nucleus, and projections to the inferior colliculus. J. Neurosci. 9:1213-1222, 1989.

MoORE DR, IRVINE DRF. Plasticity of binaural interaction in the cat inferior colliculus. Brain Res. 208:198-202, 1981.

MoORe DR, KITZES LM. Projections from the cochlear nucleus to the inferior colliculus in normal and neonatally cochlea-ablated gerbils. J. Comp. Neurol. 240:180-195, 1985.

NORDEEN KW, KILLACKEY HP, KITZES LM. Ascending auditory projections to the inferior colliculus in the adult gerbil, Meriones unguiculatus. J. Comp. Neurol. 214:131-143, 1983a.

NordeEn KW, Killackey HP, Kitzes LM. Ascending projections to the inferior colliculus following unilateral cochlear ablation in the neonatal gerbil, Meriones unguiculatus. J. Comp. Neurol. 214:144-153, 1983b.

PASIC TR, Rubel EW. Rapid changes in cochlear nucleus cell size following blockade of auditory nerve electrical activity in gerbils. J. Comp. Neurol. 283:474-480, 1989.

Pillsbury HC, Grose JH, Hall JW. Otitis media with effusion in children: binaural hearing before and after corrective surgery. Arch. Otolaryngol. Head Neck Surg. 117:718-723, 1991.

POWELl TPS, ERULKAR SD. Transneuronal cell degeneration in the auditory relay nuclei of the cat. J. Anat. 96:249-268, 1962.

Rubel EW, Hyson RL, Durham D. Afferent regulation of neurons in the brain stem auditory system. J. Neurobiol. 21:169-196, 1990.

Rubel EW, SMITH ZDJ, STEWARd O. Sprouting in the avian brainstem auditory pathway: Dependence on dendritic integrity. J. Comp. Neurol. 202:397-414, 1981.

RYAN AF. Hearing sensitivity of the mongolian gerbil, Meriones unguiculatus. J. Acoust. Soc. Am. 59:1222-1226, 1976.

Ryugo DK, Rosenbaum BT, Kim PJ, Niparko JP, SaAda AA. Single unit recordings in the auditory nerve of congenitally deaf white cats: morphological correlates in the cochlea and cochlear nucleus. J. Comp. Neurol. 397:532-548, 1998.

Sanes DH, Constantine-Paton M. The sharpening of frequency tuning curves requires patterned activity during development in the mouse, Mus musculus. J. Neurosci. 5:1152-1166, 1985a.

Sanes DH, Constantine-Paton M. The development of stimulus following in the cochlear nerve and inferior colliculus of the mouse. Dev. Brain Res. 22:255-267, 1985b.

Sanes DH, Siverls V. Development and specificity of inhibitory terminal arborization in the central nervous system. J. Neurobiol. 22:837-854, 1991.

SHERMAN SM, SPEAR PD. Organization of visual pathways in normal and visually deprived cats. Physiol. Rev. 62:738-855, 1982. 
SHOre SE, VASs Z, Wys N, Altschuler RA. The trigeminal ganglion innervates the auditory brainstem. J. Comp. Neurol. 419:271285, 2000.

SIE KCY, RUBEL EW. Rapid changes in protein synthesis and cell size in the cochlear nucleus following eighth nerve activity blockade or cochlea ablation. J. Comp. Neurol. 320:501-508, 1992.

Silverman MS, ClOPTON BM. Plasticity of binaural interaction. I. Effect of early auditory deprivation. J. Neurophysiol. 40:1266$1274,1977$.

Smith ZDJ, GRAY L, Rubel EW. Afferent influences on brainstem auditory nuclei of the chicken: N. laminaris dendritic length following monaural conductive hearing loss. J. Comp. Neurol. 220:199-205, 1983.

Tierney TS, Russell FA, MOORE DR. Susceptibility of developing cochlear nucleus neurons to deafferentation-induced death abruptly ends just before the onset of hearing. J. Comp. Neurol. 378:295-306, 1997.

TRUNE DR. Influence of neonatal cochlear removal on the development of mouse cochlear nucleus: I. Number, size, and density of its neurons. J. Comp. Neurol. 209:409-424, 1982a.

TRUNE DR. Influence of neonatal cochlear removal on the development of mouse cochlear nucleus: II. Dendritic morphometry of its neurons. J. Comp. Neurol. 209:425-434, 1982b.

Trune DR, Kiessling AA. Decreased protein synthesis in cochlear nucleus following developmental auditory deprivation. Hear. Res. 35:259-264, 1988.

Trune DR, MORGAN CR. Stimulation-dependent development of neuronal cytoplasm in mouse cochlear nucleus. Hear. Res. 33:141-150, 1988a.

TRUNE DR, MORGAN CR. Influences of developmental auditory deprivation on neuronal ultrastructure in the mouse anteroventral cochlear nucleus. Dev. Brain Res. 42:304-308, 1988 b.

Tucci DL, Born DE, Rubel EW. Changes in spontaneous activity and CNS morphology associated with conductive and sensorineural hearing loss in chickens. Ann. Otol. Rhinol. Laryngol. 96:343350, 1987.

Tucci DL, Cant NB, Durham D. Conductive hearing loss results in a decrease in central auditory system activity in the young gerbil. Laryngoscope 109:1359-1371, 1999.

Tucci DL, Rubel EW. Afferent influences on brain stem auditory nuclei of the chicken: Effects of conductive and sensorineural hearing loss on n. magnocellularis. J. Comp. Neurol. 238:371$381,1985$.

WEBSTER DB. Auditory neuronal sizes after a unilateral conductive hearing loss. Exp. Neurol. 79:130-140, 1983a.

WEBSTER DB. A critical period during postnatal auditory development of mice. Int. J. Pediatr. Otorhinolaryngol. 6:107-118, $1983 \mathrm{~b}$.

WEBSTER DB. Late onset of auditory deprivation does not affect brainstem auditory neuron soma size. Hear. Res. 12:145-147, 1983c.

WEBSTER DB. Conductive hearing loss affects the growth of cochlear nuclei over an extended period of time. Hear. Res. 32:185192, 1988.

Webster DB, Webster M. Neonatal sound deprivation affects brain stem auditory nuclei. Arch. Otolaryngol. Head Neck Surg. 103:392-396, 1977.

Webster DB, Webster M. Effects of neonatal conductive hearing loss on brain stem auditory nuclei. Ann. Otol. 88:684-688, 1979.

Wilmington D, GRAY L, JAHRSDOERFER R. Binaural processing after corrected congenital unilateral conductive hearing loss. Hear. Res. 74:99-114, 1994.

WONG-RILEY MTT. Cytochrome axidase: An endogenous metabolic marker for neuronal activity. Trends Neurosci. 12:94-101, 1989.

Wong-Riley MTT, MERZENICH MM, LEAKE PA. Changes in endogenous enzymatic reactivity of DAB induced by neuronal inactivity. Brain Res. 141:185-192, 1978.

Wong-Riley MTT, Walsh SM, Leake-Jones PA, Merzenich MM. Maintenance of neuronal activity by electrical stimulation of unilaterally deafened cats demonstrable with cytochrome oxidase technique. Ann. Otol. 90(suppl 82):30-32, 1981.

WOOLF NK, RYAN AF. The development of auditory function in the cochlea of the mongolian gerbil. Hear. Res. 13:277-283, 1984.

WOOLF NK, RYAN AF. Ontogeny of neural discharge patterns in the ventral cochlear nucleus of the mongolian gerbil. Brain Res. 17:131-147, 1985 . 